\title{
Conservative therapy options for the treatment of coxarthrosis in the early stage of the condition.
}

\author{
Petya Kasnakova ${ }^{1}$, Stanislava Ivanova ${ }^{2}$, Kalin Ivanov $^{2}$, Elina Petkova-Gueorguieva ${ }^{3}$, Stanislav \\ Gueorguiev $^{3}$, Vasil Madzharov ${ }^{3}$, Anna Mihaylova ${ }^{1}$, Penka Petleshkova ${ }^{*}$ \\ ${ }^{1}$ Medical University of Plovdiv, Medical College, Bulgaria \\ ${ }^{2}$ Department of Pharmacognosy and Pharmaceutical Chemistry, Faculty of Pharmacy, Medical University of Plovdiv, \\ Bulgaria \\ ${ }^{3}$ Department of Pharmaceutical Sciences, Faculty of Pharmacy, Medical University of Plovdiv, Bulgaria \\ ${ }^{4}$ Department of Obstetrics and Gynecology, Medical University Plovdiv, Plovdiv, Bulgaria
}

\begin{abstract}
Aim: Arthrosis of the hip joint is a condition that is becoming more and more common-it affects $1 \%$ of the population and constitutes a serious problem for their working efficiency and active way of life. The deforming arthrosis limits the functional capacity of the affected patients, worsens their quality of life and leads to incapacitation of the patients. The treatment of patients with coxarthrosis has to be planned and carried out comprehensively, in accordance with the pathological findings, the stage, duration and individual abilities of each patient. In the early stages of the condition, conservative therapy plays an essential role in the prophylaxis of complications and in slowing down the process. Physical and rehabilitation methods, in combination with medicamentous therapy, yield good results.

This article focuses on the application of the comprehensive effect of conservative physiotherapeutic methods and complementary drug therapy in the early stage of coxarthrosis. The aim of the study is to investigate the efficiency of the combined application of physiotherapeutic electrical procedures and kinesitherapy methods and means in the treatment of coxarthrosis in the initial stage.

Material and methods: 18 out-patients with coxarthrosis at an average age of 68 , were subjected to a treatment schedule including: interferential current, low-frequency magnetic field, ultra phoresis with non-steroid anti-inflammatory medications, followed by a program of remedial exercise administered in 2 courses of $7 \mathrm{~d}$ at 'St. Panteleymon' Hospital in Plovdiv, in the period from September 2017 to May 2018.

Results: The range of motion in terms of degrees exhibits a tendency towards increasing at the end of the course of therapy, as compared to that at the beginning of the course. The results from the initial and final muscle tests of the hip joint showed an improvement of the muscle function (from a degree of 3.50 to a degree of 4.50).

Conclusion: In patients with coxarthrosis in the initial stage, the pain subsides after the physical and rehabilitation therapy administered in combination with medications.
\end{abstract}

Keywords: Coxarthrosis, Conservative therapy.

Accepted on July 17, 2018

\section{Introduction}

Coxarthrosis is a form of osteoarthrosis which affects the hip joints. It is the most common joint disease, of chronic nature and non-inflammatory origin, the underlying cause of which is primary degeneration [1]. This condition affects patients of working age. According to data of WHO (the World Health Organization) for 2017, the percentage of affected male and female patients is approximately the same, the male patients being slightly in the majority at $56 \%[2]$.
The hip joint has its anatomical and physiological specific features which make it susceptible to developing pathomorphological changes typical of osteoarthrosis. The etiology of the condition is associated with the processes of ageing; traumas to the joint, repetitive overstrain [3]. The main degenerative changes affect the cartilage of the joint, capsular fibrosis is developed and osteophytes are formed along the periphery of the surface of the joints. Degenerative changes are established most often in the most burdened areas of the surfaces of the joint [4]. 
Coxarthrosis, in rare cases, can be primary, idiopathic, but most often secondary, after a congenital luxation of the hip joint, Perthes disease, necrosis of the head of the femur, and post-traumatic following fractures of the acetabulum roof, etc. $[5,6]$.

The causes for the scuffing the hip joint are most often congenital dysplasia or acquired deformations of the joints, the reduction of bone density, slowing down or preventing the natural restoration processes of tissues in elderly age, overweight and obesity, excessive physical strain during work or sport, monotonous movements causing repetitive microtraumas, immobilization which restricts the flow of the synovial fluid and the transfer of nutrients to the cartilage making regeneration impossible, diseases such as diabetes, gout, inflammatory diseases, etc. [7].

Dystrophic degenerative processes take place in the femoral head; blood circulation is labored, eventually resulting in coxarthoris. The underlying cause of the condition is the discrepancy between the strain on the joint and its capacity to withstand such strain. The cartilage is an absorber that facilitates the mechanics of movements and motion, but under the influence of the factors referred to above, the cartilage becomes scuffed and worn in varying degrees, atrophies and the remaining parts are ossified [8].

In cases of coxarthrosis, several clinical symptoms are manifested, which, in combination with diagnostic images, allow for the diagnosis to be established. The clinical symptoms include pain, restricted motion of the joint, contractions and hobbling. The X-ray is quite typical-the joint slit is often narrowed, there is osteophytosis is detected on the upper end of the acetabulum. There is sub-chondral osteosclerosis, and often cysts in the bone substance of the femoral head and the acetabulum $[9,10]$.

Pain is the first sign of coxarthrosis. It appears during walking or physical strain deep in the area of the groin folds (Holevich et al.) $[10,11]$. Initially, the pain subsides during rest and relaxation, but as the condition progresses, the pain becomes persistent. The whole inner side of the thigh down to the knee may feel sore and painful. The function of the joint is disturbed not only by the pathological process but also by the typical muscular imbalance: shortening of the flexor and adductor muscles, and weakening of the abductor muscles. The extensors and rotators are also affected [12]. The balance between the muscle groups is disturbed. This makes the symptom of Trendelenburg and Duchenne evident, and results in the so called 'gluteus medius' gait. The Patrick test is positive [13]. The imbalance is established through a functional rehabilitation assessment.

The deformations of the joint result in the shortening of the leg. Together with the subconscious protection of the affected leg, a serious limp gradually appears which, at a certain point in time leads to inability to move without aids, such as walking sticks and crutches.

The flexibility of the joint gradually becomes more limited, the joint capsule contracts, and so do the muscles attached to it, which results in a non-arbitrary bending of the leg, its being drawn to the other one, and its inability of turn inwards.

The comprehensive therapy of osteoporosis of the hip joint is aimed at: relieving the pain syndrome, maintaining the functionality of the joint, preventing and remedying joint deformations, slowing down the destructive process. The first imperative step in the medical treatment is the elimination of all aspects and factors leading to excessive straining of the joint [12].

Depending on the X-ray data and clinical symptoms, three stages of coxarthrosis can be differentiated $[4,7]$.

The initial stage of coxarthrosis (pre-coxarthrosis) may last for up to several years. The condition progresses with remissions and exacerbations, and this determines the strategy of the medicamentous and physical therapy. In the early stages of the condition, conservative therapy is administered. Physical methods of rehabilitation are considerably efficientphysiotherapeutic procedures, as well as spa and balneological treatment. Kinesitherapy procedures and massage are important for maintaining muscle tone, optimizing blood flow to the joint structures, maintaining and increasing the range of motion [14].

The selection of means and methods is customized, tailored to the condition of each patient, ad gradual, compliant with the functional test data. A summarized clinical algorithm of the process of physical rehabilitation and re-education includes: kinesitherapy-specialized and highly specialized methods, physiotherapeutic schedule of pre-formed and natural physical factors, training the patient to walk.

Kinesitherapy is a major component of the conservative treatment of coxarthrosis. When applied consistently and continuously, combined with an individual approach to each patient, it is quite efficient and yields good results in the treatment of the condition. After performing the respective tests needed to determine the current condition each patient (a snapshot of the patient's condition), a kinesitherapeutic treatment schedule is elaborated. If the affected joint is further burdened by overweight or an inappropriate job, these factors have to be eliminated for the purpose of slowing down the progress of the condition. The kinesitherapy tasks, methods and means vary depending on the stage of the disease [15].

In the initial stage, the main tasks and aims are connected with: maintaining the functionality of the hip joint, improving circulation, trophics and lymph flow, restoring muscle balance, developing correct posture and gait, correcting the deformation, releasing and relieving the hip joint using aids, prophylaxis of complications and contractures, enhancing the overall condition and tone of the whole body, reducing body weight.

The following kinesitherapy means and methods are used: medical massage, zone therapy, apparatus massage, exercises aimed at generally strengthening the body, combined with respiratory exercises, relaxation, exercises in suspension, exercises for the unaffected limb aimed at reducing resistance, 
post-isometric relaxation exercises, proprioceptive neuromuscular facilitation methods of relaxation, analytical exercises done from a relieved initial position, exercises for the abdominal wall and paravertebral muscles, isometric contractions, exercises aimed at improving the gait/way of walking/and improving the balance, manual mobilization of the hip joint, stretching and extension exercises, exercises underwater, therapy implemented while the patient is lying in a hard mattress or mat, exercises aimed at facilitating the performance of everyday tasks and chores[16].

Exercises that put an additional strain on the hip joint are contra-indicative.

\section{Training how to walk}

Coxarthrosis patients have to be trained how to walk correctly, but this has to be done slowly and gradually, because patients unconsciously try to spare the affected leg. The training has to continue until the gait of the patient becomes symmetrical. Unfortunately, this has proven to be very difficult to achieve because any change in the dynamic locomotor process requires strong will, persistency and time for the learned to become automatic.

\section{Physical therapy}

Physiotherapy plays a leading role in the early stages of the condition.

The following physiotherapeutic methods are used for pain relief: electrophoresis and diadynamophoresis with lidocaine $2 \%, 10 \mathrm{ml}$ solution for injection and novocaine solution for injections $2 \%$, low-frequency currents in the pain-relief range and TENS-therapy, sinusoidally modulated currents of the $3^{\text {rd }}$ and $4^{\text {th }}$ group-modulation depth $25 \%$ and a frequency of 100 $\mathrm{Hz}$; interferential currents-90-100 Hz-for pain suppression [13].

The following are efficient in trophic improvement: centimeter waves, decimeter waves, low-impulse magnetic field, electrophoresis or ultra phonophoresis with non-steroid antiinflammatory medications. All these are aimed at slowing down the aging of the cartilage, preventing the joint capsule from fibrosis, and facilitating the spread of the residual synovitis; in terms of thermal therapy, the following are highly beneficial: psalm therapy, lye and paraffin applications. In cases of weakened $\mathrm{m}$. quadriceps femoris, electrical stimulation with low-frequency currents-rectangular-shaped impulse, frequency 20-50 Hz-are applied [13].

Conservative therapy is mainly focused on enhancing the restorative capacity of the organism.

From the early stages of the condition, it is important that the patient should commence chondroprotective therapy (glucosamine, chondroitine, hyaluronic acid, collagen, etc.) so that the process of degradation of the hyaline cartilage can be slowed down. Non-steroid anti-inflammatory medicines with an analgesic and anti-inflammatory effect, as well as analgesics with central effect are used to relieve the medical symptoms. In cases of expressed muscle contracture, myorelaxants are recommended and administered. Glucocorticosteroid medicines may be applied for a short period of time, even in the joint itself, but only in cases of expressed inflammatory activity of the arthouses process, with particular care. Muscle relaxants are also needed to help muscle tissue relax in cases of coxarthrosis. The relief of the muscle spasm helps relieve the pain experienced by the patient $[7,8]$.

At the beginning and at the end of the process of treatment, clinical, para-clinical and instrumental assessment of the condition of the patient is made so as to evaluate the results from the administered physical therapy and rehabilitation. When the different therapeutic procedures are combined correctly, and synergism between the physical and medicamentous therapy is achieved, we observe and have objectivized many times reliable favourable results.

\section{Our own observations}

The aim of the study is to investigate the efficiency of the combined application of physiotherapeutic electrical procedures and kinesitherapy methods and means in the treatment of coxarthrosis in the initial stage.

\section{Material and Methods}

Eighteen out-patients with coxarthrosis at an average age of 68 $\mathrm{y}$, were subjected to a treatment schedule including: interferential current, low-frequency magnetic field, ultra phoresis with non-steroid anti-inflammatory medications, followed by a program of remedial exercise administered in two courses of $7 \mathrm{~d}$ at 'St. Panteleymon' Hospital in Plovdiv, in the period from September 2017 to May 2018. All patients included in the survey had had complaints of pains and stiffness for over a year. The average value of the period of having pains was 16 months. The criteria for including patients in the study were determined in accordance with the functional principle, and included exacerbating pain and restricted range of motion. Patients in the second or third stage of the condition, or after endoprosthetic replacement, were not included.

The pain was reported according to the visual-analogue scale prior to each procedure. The range of motion of the hip joint was measured using a standard angle-gauge at the beginning and at the end of the course of therapy. Also, at the beginning and at the end of the course of treatment, the status of the weakened muscles is assessed through manual muscle testing.

\section{Results}

The pain started to subside significantly after the fourth procedure of the first course of procedures, and afterwards continued to decrease statistically until the end of the second physiotherapeutic course. The patients themselves assessed the degree of the pain they experienced during movement (Figure 1). 
The range of motion, expressed in grades, shows insignificant tendency towards an increase at the end of the first course of procedures, however, after the second course of procedures combined with auxiliary kinesitherapy procedures at home, we observed a significant effect in the mobility and flexibility restoration of the affected hip joint (Table 1).

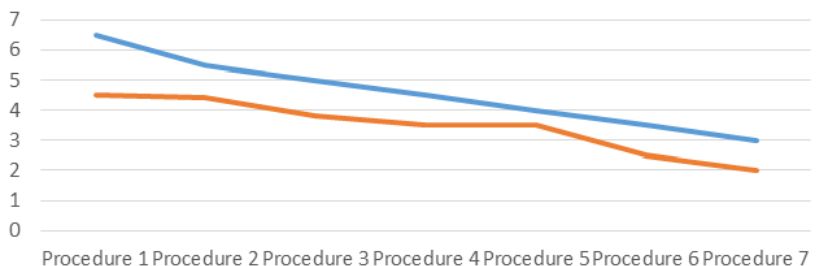

-1st Course

Figure 1. Comparative study into the changes in the values of the pain according to the visual-analogue pain assessment scale (VAS).

Table 1. Results from the performed anglemetry aimed at investigating the active range.

\begin{tabular}{llll}
\hline \multirow{2}{*}{$\begin{array}{l}\text { Movement of the } \\
\text { hip joint }\end{array}$} & \multicolumn{3}{l}{ Research into the range of motion } \\
\cline { 2 - 4 } & $\begin{array}{l}\text { At the beginning of } \begin{array}{l}\text { At the end of the } \\
\text { course } \\
\text { treatment }\end{array} \\
\text { course } \\
\text { treatment }\end{array}$ & $\begin{array}{l}\text { In } \\
\text { months }\end{array}$ \\
\hline Abduction & $10,25^{\circ}$ & $25,50^{\circ}$ & $30,25^{\circ}$ \\
\hline Extension & $5,50^{\circ}$ & $10,25^{\circ}$ & $20,00^{\circ}$ \\
\hline Flexion & $55,25^{\circ}$ & $75,50^{\circ}$ & $85,25^{\circ}$ \\
\hline Internal rotation & $15,25^{\circ}$ & $20,25^{\circ}$ & $30,50^{\circ}$ \\
\hline
\end{tabular}

The range of motion in terms of degrees exhibits a tendency towards increasing at the end of the course of therapy, as compared to that at the beginning of the course. The effect of the used analytical kinesitherapy methods aimed to increase the abduction in the hip joint proved to be good, as shown in the final tests, reaching $30^{\circ}$. The values for extension prior to the therapy indicate deficit, but after the therapy, in particular after the $6^{\text {th }}$ month, a considerable improvement is observed, reaching $20^{\circ}$.

Internal rotation did now show a substantial deficit limiting the functional capacity of the lower extremity, which called for shifting the focus to the restoration of this motion. The external rotation values were within normal limits during that period.

The dynamics of change in the values of the parameters marked a permanent improvement in the period under review in the range of motion of the hip joint.

The results from the initial and final muscle tests of the hip joint showed an improvement of the muscle function (from a degree of 3.50 to a degree of 4.50). Improvement of the muscle strength was observed in all patients, especially in the strength of the extensors and abductors, which are the main muscles involved in the final support phase during walking.

\section{Discussion}

The administered electrotherapy with interferential current, low-frequency magnetic field, ultra phonophoresis with nonsteroid anti-inflammatory medications, has a short-term symptomatic effect; the pain, however, subsides considerably as early as after the third procedure. The combined physiotherapy and rehabilitation treatment have a good effect on the surveyed patients. The subsiding of the pain substantially improves the range of motion, and therefore the muscle balance is restored. As a result of including specialized analytical kinesitherapy methods, muscle strength and resilience are improved, as evidenced by the improved statics and gait. The positive results of the comprehensive treatment of the patients-subjects in the study-may are accounted for by the specific effects of such an approach. During the whole period of the study, the patients tolerated the procedures quite well, and no adverse local or overall reactions were reported.

\section{Conclusion}

In patients with coxarthrosis in the initial stage, the pain subsides after the physical and rehabilitation therapy administered in combination with medications. The combination of specialized rehabilitation methods lead to an increase in the mobility of the hip joints, which, in turn, leads to improved stability while walking.

The treatment and physical rehabilitation of such patients require serious medical-social procedures and actions, which result in an improved quality of life for the patient, as well as to his/her improved emotional and mental adaptation. Coxarthrosis leads to degenerative changes in the hip joint and the para-joint tissues, and hence to impairment of the locomotor function of all groups involved in walking and standing. The therapy depends on the stage at which the condition is detected. When the condition is detected in a more advanced stage, the therapy should be more careful, better planned, and, at the same time, more intensive.

\section{References}

1. Felson DT. Epidemiology of osteoarthritis, in ostheoarthritis (2nd ed.). Oxford Univ Press, Oxford 2007.

2. World Health Organization. International statistical classification of diseases and related health problems. WHO 2018; 1.

3. Popov N, Popova D, Grueva T. Physiotherapy in musculoskeletal dysfunctions of the lower limbs. NSA Press, Sofia 2003.

4. Hep V, Debbrunner X. Orthopedic diagnostics. Med Phys Ed, Sofia 2010.

5. Williams M, Frankel S, Nanachal K, Coast J. Total hip replacement: epidemiologically based needs assessment. Health Care Evaluation Unit, Univ Bristol, Bristol 1992. 
6. Bankov S, Krasteva V, Vajarov Y. Manually muscle testing with the basics of kinesiology and pathogenesisology. Sofia Med Phys Ed 1987.

7. Stavrev P. Orthopedics and traumatology. Elsevier 2004.

8. Poitout D, Gaujoux G. Osteoarthritis of the hip. Aetiology, pathophysiology, diagnosis, principles of management. EULAR Bulletin 1991; 3: 82-88.

9. Kostadinov D. Coxarthrosis. Med Phys Ed, Sofia 2011.

10. Kostadinov D, Nikolova L, Bankov S. Physical factors in the complex treatment and rehabilitation of some common diseases. Me Phys Ed, Sofia 1980.

11. Popova D. Hip joint happens. In Functional Studies and Analysis of Musculoskeletal Physiotherapy. NSA Press 2012; 229-225.

12. Karaneshev G. Theory and methodology of the physical education. Sofia 1991.

13. Ryakova M, Kirova I, Aleksiev A. Practical clinical physiotherapy. Know 1999.
14. Zhelev V. Post-isometric relaxation by K. Levit. In Physiotherapy (Third part). Avangard Prima 2011; 228-230.

15. Krajjikova L. Soft-tissue hip mobilization techniques for coxarthrosis. Kinesitherapy Rehabilitation 2009; 3: 43-45.

16. Bennell K. Physiotherapy management of hip osteoarthritis. J Physiother 2013; 59: 145-157.

\section{*Correspondence to}

Penka Petleshkova

Department of Obstetrics and Gynecology

Medical University Plovdiv

Plovdiv

Bulgaria 